\title{
Consequence of midline mandibular flexure on bilaterally splinted and non-splinted implant-supported mandibular full arch prosthesis with immediately loaded implants: A one year clinical study
}

\author{
Mostafa Helmy Mostafa Ahmed ${ }^{1}$, Henri Diederich ${ }^{2 *}$ and Mohamed M Abo Heikal ${ }^{3}$ \\ ${ }^{1}$ Lecturer of Removable Prosthodontics/ Faculty of Dentistry, Cairo University, Egypt \\ ${ }^{2}$ Henri Diederich Dental Clinic, 51 as Pasteur, L- 231, Luxembourg \\ ${ }^{3}$ Assistant lecturer of Removable Prosthodontics/ Modern Science and Arts University, Egypt
}

\begin{abstract}
Objectives: The aim of this study was to compare the effect of the mandibular deformation by medial flexure on Bilaterally Splinted and Non-Splinted ImplantSupported Mandibular Full Arch Prosthesis with Immediately Loaded Implants both clinically \& radiographically. In addition to Patients' satisfaction over one year of clinical performance.

Materials \& methods: For the sake of clinical investigation, twelve completely edentulous patients obtained new mandibular and maxillary complete dentures before implant placement. All patients were educated to use the denture for two months. After two months; patients were indiscriminately divided into two groups; First group (of six patients) obtained an Implant supported, fully-splinted cement-retained full arch prosthesis with immediate functional loading protocol, while Second group (of six patients) obtained an Implant-supported, non-splinted (segmented) cement-retained full arch prosthesis with immediate functional loading protocol. The clinical \& the radiographic outcomes of the Implants supporting the cement retained full arch superstructure, had been calibrated at time of implants insertion, 3 months, 6 months and 12 months respectively. In addition, patients' satisfaction was also measured utilizing a customized chart of question (A seven-point scale)

Results: regarding parametric data; repeated gauges ANOVA test was used to compare between the two groups as well as to study the changes by time within each group. Bonferroni's post-hoc test was used for pair-wise comparisons when ANOVA test is significant. whereas for non-parametric data; Mann-Whitney U test was utilized to compare between the two groups. Periotest and bone density data showed normal (parametric) distribution while bone loss and satisfaction scores data showed non-normal (non-parametric) distribution.

Conclusion: Regarding effect of midline mandibular flexure on bilaterally splinted \& nonsplinted(segmented) cement-retained, immediately-loaded mandibular prosthesis, it was found that, there was non-significant effect on both types of prosthesis and hence, splinted or nonsplinted implant-supported prostheses could be used successfully with little superiority of segmentation in midline region. In addition, Patients were completely satisfied with their implant-supported immediatelyloaded prostheses, whatever splinting protocol utilized.
\end{abstract}

\section{Introduction}

Mandibular flexure is defined as "the change in shape of the mandible caused by the pterygoid muscles contracting during opening and protrusion movements" [1]. Where, it may affect the biomechanical behavior, passive fit, and prognosis of the fixed implant-supported prosthesis if it is not put into consideration [2].

Data analysis of the mechanical properties of the human mandible is difficult because there is a large variation in mandibular size and bone density in the population.

In natural dentition, the stress generated by mandibular flexure can be compensated by adaptation of the periodontal ligament. While, these stresses in a fixed implant-supported prosthesis are transmitted around the mandibular bone and could induce stresses in the implantrelated prosthesis and abutments and hence, affecting the bone-implant interface [3, 4], especially in posterior implants [5]. Consequently, mandibular flexure should be considered an important biomechanical factor in the design of a partial or complete fixed implant-supported prosthesis in the mandible with dental implants [6-8].

The term medial mandibular flexure (MMF) refers to mandibular deformation characterized by a decrease in arch width during jaw opening and protrusion movements because of the functional contraction of the lateral pterygoid muscles, the muscle action during these movements makes both mandibular rami approach. In other words, there is a reduction of the intercondylar distance causing high strain in the symphysial region $[9,10]$.

Correspondence to: Henri Diederich, Henri Diederich Dental Clinic, 51 as Pasteur, L- 231, Luxembourg, Email: hdidi@pt.lu

Key words: mandibular flexure, splinting, deformation, immediate loading, patient satisfaction

Received: March 12, 2018; Accepted: March 28, 2018; Published: March 31, 2018 
Ahmed MHM (2018) Consequence of midline mandibular flexure on bilaterally splinted and non-splinted implant-supported mandibular full arch prosthesis with immediately loaded implants: A one year clinical study

It would be reasonable to expect that stronger muscles would be associated with larger mandibular flexure [11]. The influence of geometric facial factors on mandibular deformation is unclear as only a few measures have been found to be statistically significant [12].

During oral rehabilitation of an edentulous patient with an implant-supported prosthesis, mandibular flexure should be considered an important biomechanical factor when planning the metal framework design, especially if implants are installed posterior to the interforaminal region [13].

The oral rehabilitation of an edentulous patient handled with a fixed implant-supported prosthesis utilizing suitable biomechanical and prosthetic fundamentals has been a target in oral implant research for the last era [14]. With the fixed implant-supported prosthesis, an adequate distribution of stress is very important to decrease implant and prosthetic failures [15]. In addition, these failures can also be influenced by several factors, including prosthetic design and occlusal scheme [16].

A common treatment strategy for an edentulous mandible is the installation of implants in the inter-foraminal region and a full-arch fixed implant-supported prosthesis with cantilever distal extensions [17-19]. However, this type of prosthesis can promote a high level of stress that can be harmful to the implant and the surrounding bone because of the unfavorable lever arms [20]. For this reason, it has been suggested that the use of multiple implants in the anterior and posterior mandible could improve the distribution of stress with more favorable implant support, avoiding long cantilevers [21].

Completely edentulous patients with adequate bone height and width can be successfully restored with fixed implant-supported prostheses. the edentulous mandible can be restored successfully with an immediately-loaded implant-supported fixed prosthesis. There are several studies about the possible consequences of bilateral rigid connection with distal extension in conventional prostheses. Some associate MMF with muscle pain, limited mouth opening, absence of prostheses passivity, among other complications [22].

With the onset of Implantology, the replacement of fixed dentures in mandible by implant-supported fixed prostheses became a reality and with it, the demand to resolve the problem of medial mandibular flexure [23].

Researchers discussed the biomechanical effects of medial mandibular flexure in the accumulation of stress in implant-supported fixed restorations. While others reported a possible correlation between the MMF and discomfort experienced by a patient rehabilitated with fullarch implant-supported fixed prostheses [24].

Regarding prosthetic considerations on implant-supported prostheses, Certain authors declared that; to compensate the medial mandibular flexure caused by the pterygoid muscle contraction, the prosthesis can be constructed in segments; thus, it does not have a rigid structure involving functional bone flexure, which could generate unfavourable stresses [25].

Many researchers believe that MMF can affect the stress distribution in implant supported fixed partial prostheses and, however, this factor has been neglected in most finite element analysis of the mandible $[26,27]$. Some clinical studies performed in the last years $[28,29]$ have demonstrated that there are three important patterns of stress and deformation during normal mandibular movements that can be measured using displacement transducers. These are corporal (bodily) rotation $(\mathrm{CR})$, corporal approximation $(\mathrm{CA})$ and dorsoventral (DV) shear.

Dorsoventral (DV) shear is the movement of the 2 halves of the mandible relative to one to another in the vertical plane $[4,8,9]$. This movement would be observed as changes in orientation of the top of the implants in the sagittal plane. While, Corporal rotation (CR) is measured determining the relative outward rotation of two implants inserted in the jaw as the corpora rotated and their coronal ends became further apart relative to their apices [30]. This rotation was related to the changes in orientation of the tops of the implants in the transverse plane. Such an approach results in deformation being expressed as an angle. And finally, Corporal approximation (CA) is the change in mandibular width during function [31]. It would be observed as a change in the orientation of the top of the implants in the horizontal plane. Earlier researches reported that jaw deformation could be a problem during impression-making; an impression taken with the mouth wide open may not fit passively in other jaw positions. The basis of concern is that deformation may result in an ill-fitting superstructure or the creation of harmful strains in the patient/implant complex [32].

Certain authors have observed that dimensional changes in the mandible occur during the maxillary activity due to the action of the masticatory muscles. The measurement of this phenomenon has been performed by different techniques, mainly; intraoral and extraoral. According to some authors there is no significant difference in medial mandibular flexure in the maximum opening between men and women, age ranges and different configurations of the mandibular arch [33]. The influence of the mandibular deformation by medial flexure on Bilaterally Splinted and Non-Splinted Implant-Supported Mandibular Full Arch Prosthesis with Immediately Loaded Implants were examined both clinically \& radiographically. In addition to Patients' satisfaction over one year of clinical functioning.

The null hypothesis was postulated; there will be non-substantial variation between both techniques all over the whole investigation phase.

\section{Materials and methods}

Twelve patients were carefully selected from the outpatient clinic of the Removable Prosthodontics department, Faculty of Oral and Dental Medicine, Cairo University. Where, patient selection was accomplished according to the following criteria:

- Age ranged between 55-65 years.

- Patients with completely edentulous arches (mandible \& maxilla). (Figure. 1)

- Patients exhibited sufficient inter-arch space (at least $15 \mathrm{~mm}$. from the mandibular ridge to the occlusal plane). - Patients with good oral hygiene.

- Patients free from bad oral habits.

- Patients free from any systemic or debilitating diseases such as diabetes mellitus, bone diseases, blood discrasis or other diseases that affect bone healing around the implants.

- Absence of any medical disorder that might obscure the surgical phase or disturb osseointegration.

- Patients with Angle's class I maxillo-mandibular relationship with normal occlusion. 
Ahmed MHM (2018) Consequence of midline mandibular flexure on bilaterally splinted and non-splinted implant-supported mandibular full arch prosthesis with immediately loaded implants: A one year clinical study

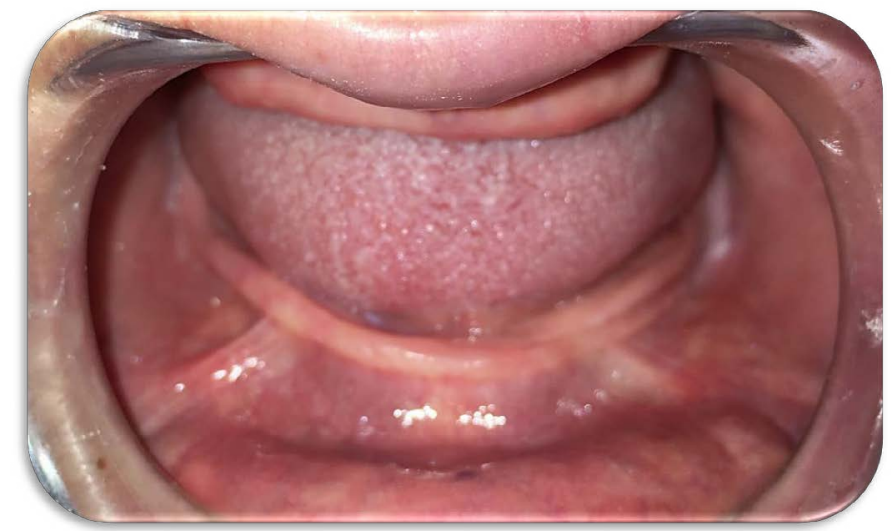

Figure 1. A patient with completely edentulous arches (mandible \& maxilla.

- Heavy smokers (more than 30 cigarettes per day), were excluded from this study.

- Uncooperative patients were omitted \& only cooperative patients were included in the study

The patients were asked for their approval to the conduction of the research \& being recalled for follow-up appointments. All details were written \& signed by the patients in consent forms.

The study was conducted according to principles stated in Helsinki Declaration \& being approved by the Faculty ethical committee.

After taking full patient's personal, medical and dental history, each patient received a thorough clinical and radiographic examination.

\section{Construction of the complete dentures prior to surgery (Figure 2)}

- Upper and lower primary impressions were made utilizing alginate impression material ${ }^{1}$ according to the manufacturer's instructions and poured into stone plaster ${ }^{2}$ to attain diagnostic casts over which acrylic resin ${ }^{3}$ special trays were fabricated.

- For every patient, the casts were mounted on a simple hinge articulator aided by a Tentative inter-occlusal wax record. Afterwards, the occlusal relation between the upper and lower teeth was thoroughly examined.

- Final impressions were made by two-step rubber base impression material ${ }^{4}$ according to the manufacturer's instructions.

- Master casts were obtained, Occlusion blocks were constructed centric jaw relation was recorded using the traditional wax-wafer method.

- Occlusion blocks on their master casts were mounted on semiadjustable articulator, where, the upper cast was mounted according to a face bow record \& the lower cast was mounted by the aid of the recorded centric jaw relation record.

- Setting-up of teeth then, Try-in stage was carried out in the usual manner. When assuring satisfactory Try-in, the dentures were completed till obtaining the final maxillary \& mandibular complete dentures following the traditional steps of construction.

1 Cavex alginate, dust free, high consistency, Holland

2 Type III dental stone Lascod SP, sestofino, Italy

3 Moldano. Bayer Leverkusen, pekatray, Germany

4 Panasil, Katzenbach, Germany
Patients were asked to utilize their dentures for two months of adaptation with a recall appointment every single week for denture assessment $\&$ any needed occlusal refinement.

It is worth to clarify that all dentures were fabricated by the same dental technician in the same laboratory.

\section{Patient imaging and case planning (Figure 3)}

- Duplication of the mandibular complete denture was encountered utilizing a radioopaque material to construct radiographic stents for every patient.

- Patients were imaged using cone beam computed tomography scans (CBCT scans) through a cone beam CT machine (CBCT, i-CAT Vision $)^{5}$. each patient was instructed to bite on a piece of cotton to achieve adequate jaw separation. Finally, the resultant image was obtained as a DICOM file.

- The images were processed using specialized image processing software (Blue Sky implant software) ${ }^{6}$.

\section{Surgical guide fabrication}

virtual implants were placed in the position of lower anterior \& posterior teeth from lower left second molar to the lower right second molar (14 implant positions), A solid block was modeled \& guiding holes denoting the implant direction were opened into the block.

\section{Pre-surgical steps}

- The surgical stent was sterilized chemically to be used during surgery.

- The patient was instructed to take a prophylactic antibiotic

\footnotetext{
5 Imaging Sciences International, Hatfield, Pa, USA 6 Blue Sky Bio, LLC
}
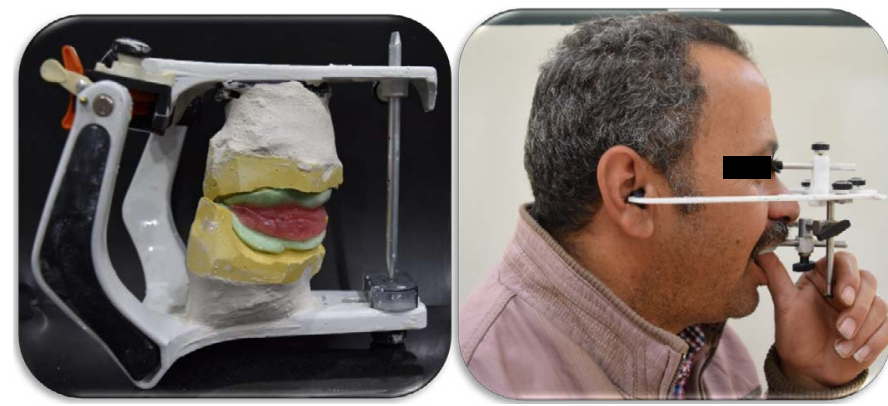

Figure 2. Pre-operative Clinical Procedures

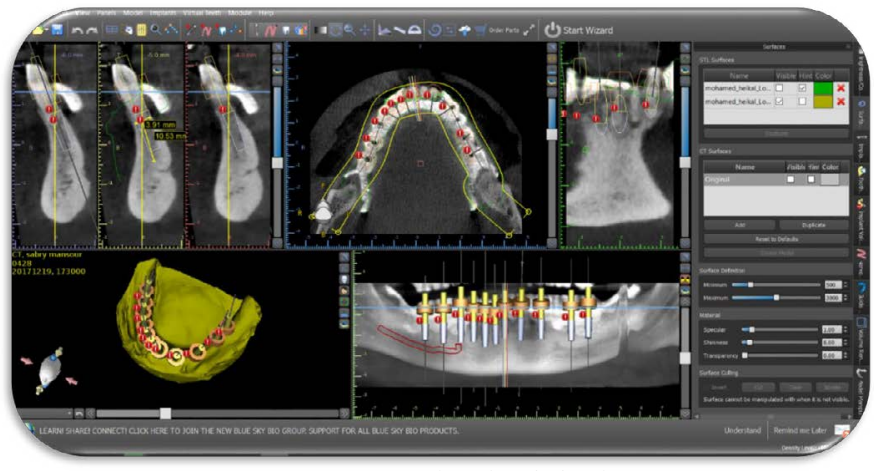

Figure 3. Pre-operative Virtual Planning. 
Ahmed MHM (2018) Consequence of midline mandibular flexure on bilaterally splinted and non-splinted implant-supported mandibular full arch prosthesis with immediately loaded implants: A one year clinical study

preoperatively ${ }^{7}$ and to rinse with chlorohexidine mouth wash ${ }^{8}$ four hours before surgery.

\section{Surgical procedures (Figures 4-8)}

Two-stage surgery was followed, and the patients could wear their dentures during the healing period in-between: First surgical phase

- The entire surgical armamentarium was autoclaved.

- The surgical place as well as the circumoral tissues were also disinfected by wiping them with antiseptic solution'. A mandibular nerve block anaesthesia was given Bilaterally, using $4 \%$ articaine anesthetic solution ${ }^{10}$. Also, field block anaesthesia was applied to diminish the bleeding as much as possible.

- The surgical template was introduced into the patient's mouth, seated over the mandible and checked for accuracy \& stability in place.

- Osteotomy sites for the implants were performed using a pilot drill of $2 \mathrm{~mm}$ diameter \& a final drill for coronal flaring.

7 Augmentin $625 \mathrm{mg}$ Beecham, MUP

8 Listerine mouthwash, USA.

9 Listerine mouthwash, USA

10 Ubestesin, 3M ESPE, Germany

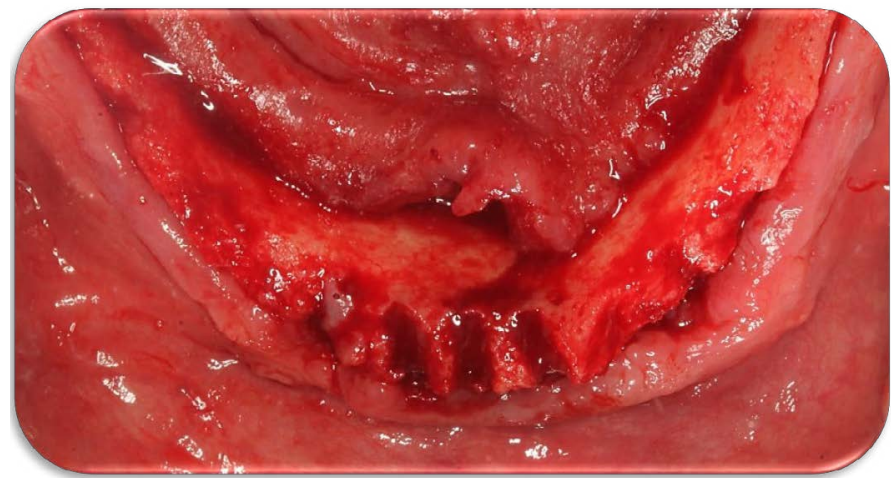

Figure 4. Incision \& Mucoperiosteal flap reflection.

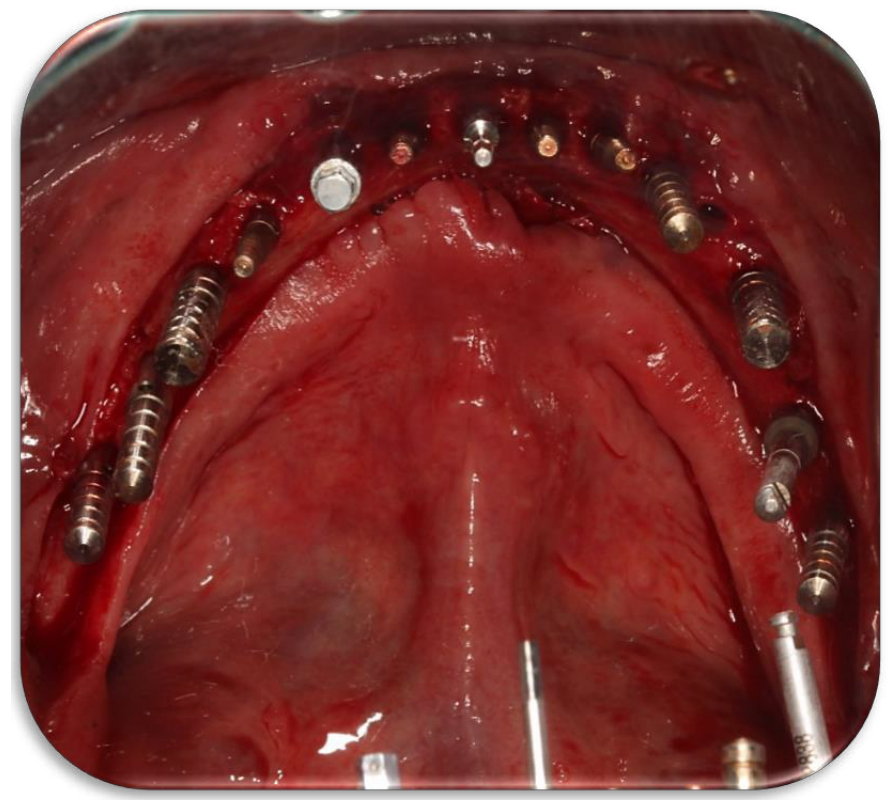

Figure 5. Parallel pins to check Parallelism

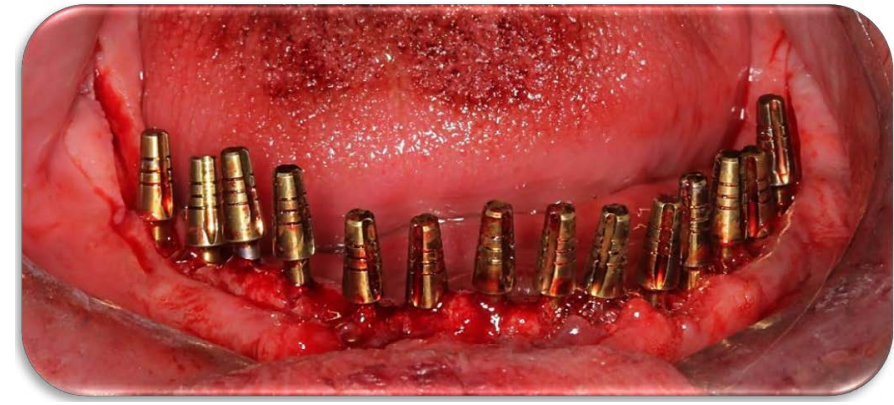

Figure 6. Proper placement of the Implants

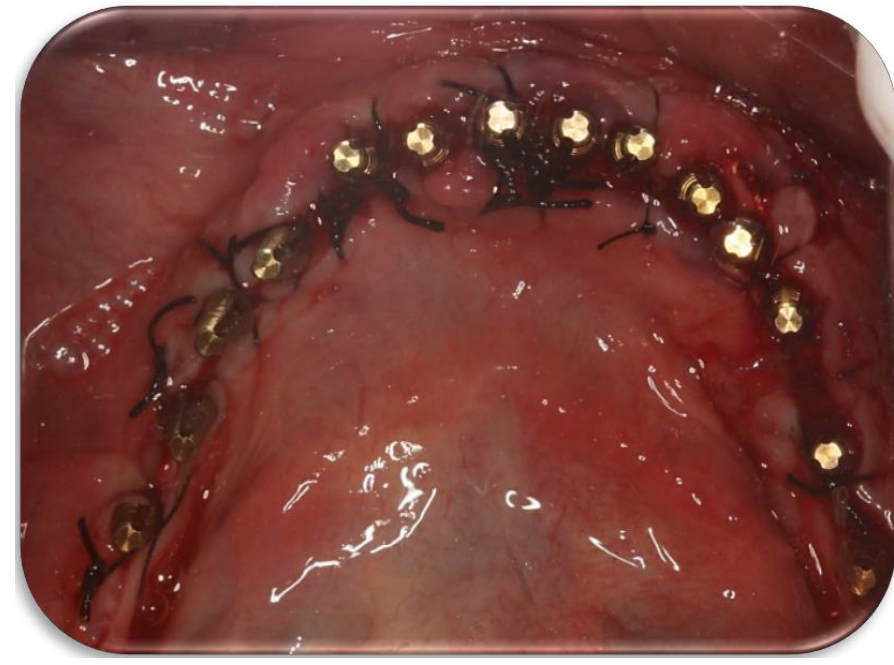

Figure 7. Suturing of the Flap

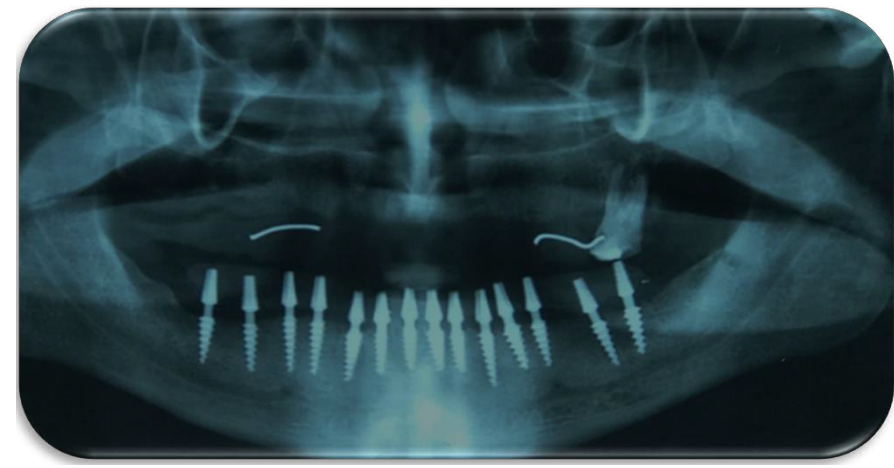

Figure 8. A Post-Operative Panoramic x-ray

\section{Implant insertion}

- The sterile box of the implant ${ }^{11}$ was unwrapped, and then the inner vial was also opened \& the implant osteotomy was washed thoroughly using sterile saline solution.

- The sterile implant was introduced into its site by screwing it using moderate finger pressure [self-tapping] Once resistance was felt, the abutment was unscrewed from the implant fixture \& the ratchet wrench was adapted to the implant and the screwing process was continued.

- The screwing process was stopped when the implant becomes flushed with the crest of the bone or preferably $0.5 \mathrm{~mm}$ below the crestal bone level.

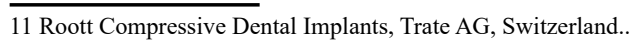


Ahmed MHM (2018) Consequence of midline mandibular flexure on bilaterally splinted and non-splinted implant-supported mandibular full arch prosthesis with immediately loaded implants: A one year clinical study

* Then, utilizing the Periotest device ${ }^{12}$, four readings (buccal, lingual, mesial \& distal) were obtained for each implant. those readings represented the first clinical readings.

Finally, a Panoramic radiographic picture was obtained for the implants to ensure proper positioning. Whereas Other separate radiographs were taken to be read out by the Digora computerized system to calculate the first radiographic readings.

\section{Post-surgical instructions}

The patients were immediately given after surgery Diclofenac Sodium non-steroidal antiinflammatory analgesic tablets (Voltaren $75 \mathrm{mg})^{13}$. It was prescribed as one tablet three times daily for three days to reduce pain and swelling and were advised to follow the antibiotic regimen previously prescribed (Augmentin $1 \mathrm{~g}$ ) for 5-7 days. Patients were given the following instructions:

- To apply ice packs for 10 minutes with 10 minutes intervals along a period of 3-4 hours immediately following surgery.

- To follow strict oral hygiene protocol.

- To avoid eating hard food for one month. (only soft diet)

- asked for some recall appointments within the next week till delivering the final restoration after three months, to complete the restorative procedures.

\section{Restorative procedures (Figures 9-25)}

Directly following implant placement surgery, impression caps were snugly fitted onto corresponding abutments, then A single-step impression (utilizing Putty and light addition silicon rubber base impression $)^{14}$ was made.

A prefabricated Acrylic Resin Stent was utilized for direct construction of a temporary prosthesis that was made completely out of occlusion (using a special temporary crown material) ${ }^{15}$, and the temporary prosthesis was then adjusted intra-orally.

Finally, A shade color selection was performed.

\section{Periotest, Sweden}

13 Voltaren, $75 \mathrm{ml}$ oral, NOVARTS, Egypt

14 Panasil, Katzenbach, Germany

15 Pentron, USA

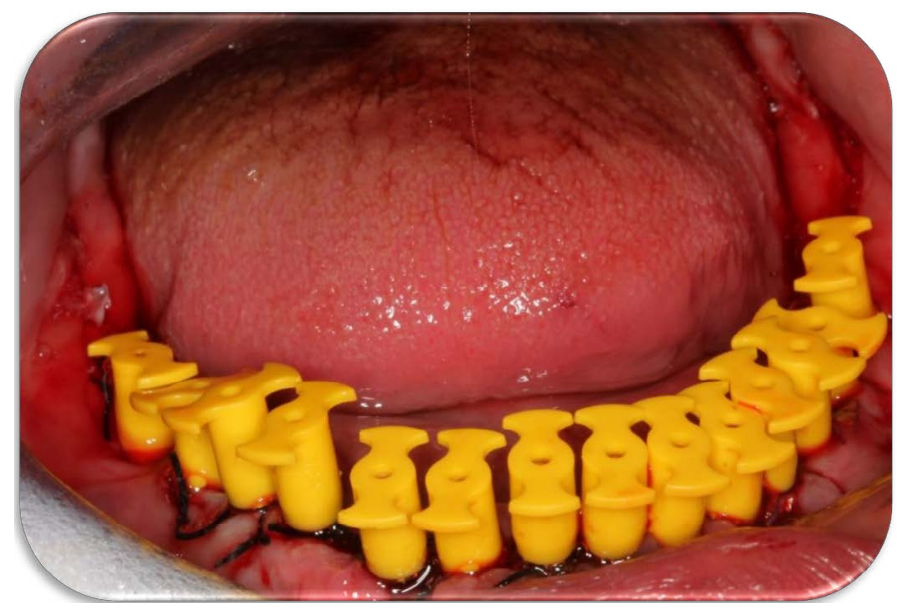

Figure 9. Placement of Impression Caps

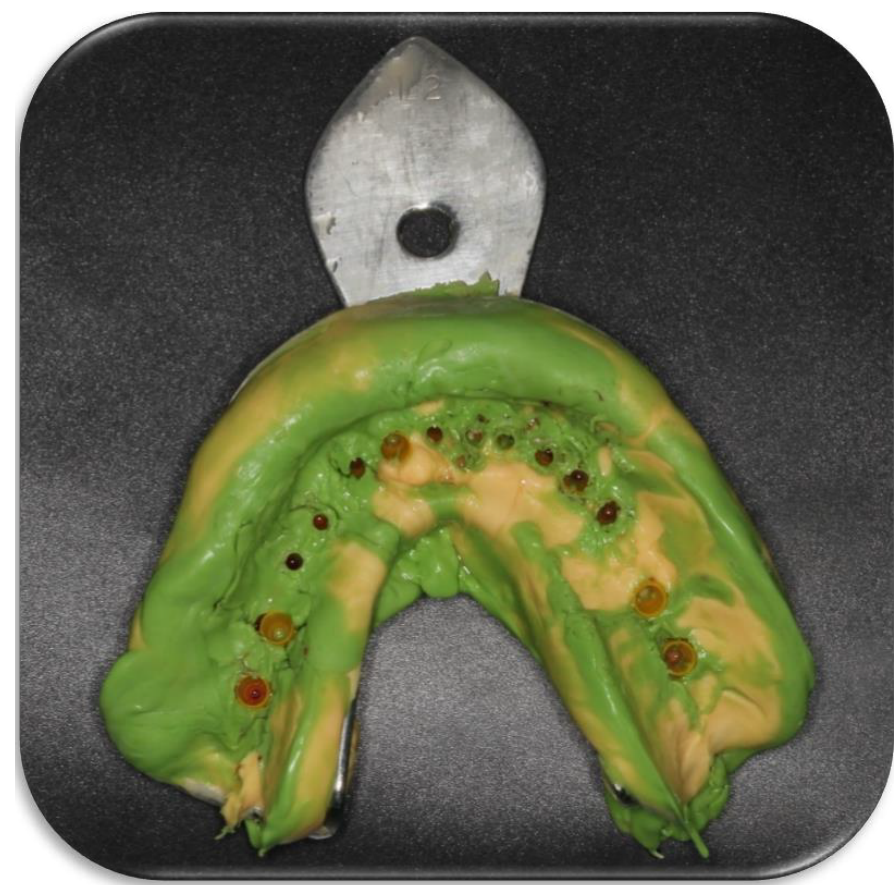

Figure 10. Single-step impression enclosing the impression caps

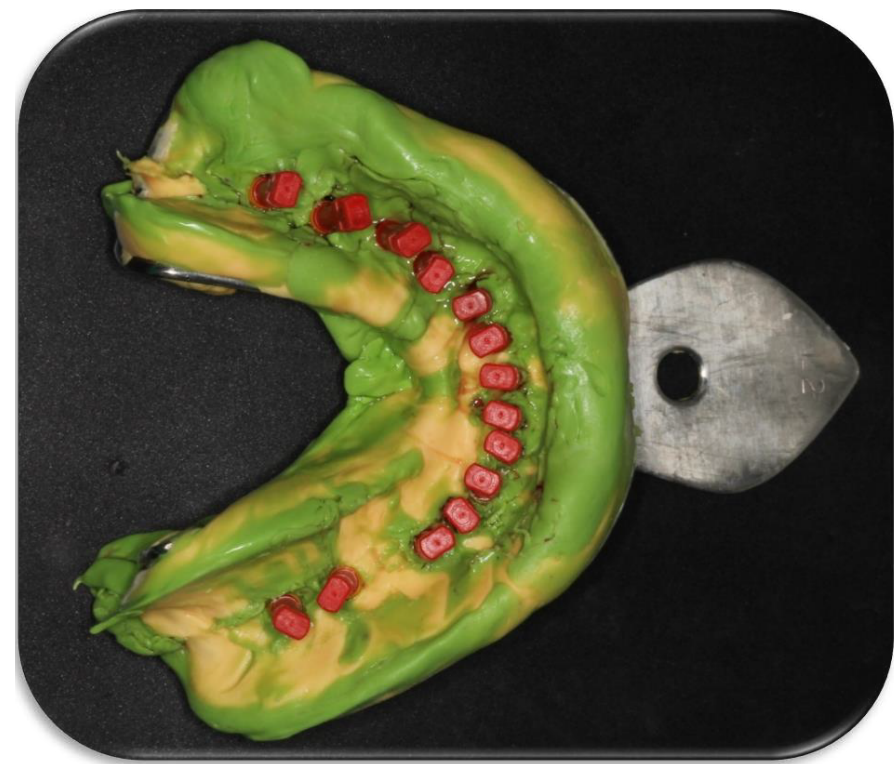

Figure 11. Analogues fitted onto their corresponding impression caps

\section{Laboratory procedures}

Single-piece plastic transfer copings suitable for the used abutments were fitted on their corresponding impression caps inside the impression surface.

In the laboratory, A small cotton pellet was utilized to varnish the impression surfaces surrounding the analogues with Vaseline. Then, a gingival mimic was created around the analogues using a plastic impression syringe loaded with a special gingival mimic material ${ }^{16}$ was performed, then impression was poured utilizing extra-hard stone to obtain a cast that enclosed the implant analogue part with attached abutment analogue were apparent from the cast.

$\overline{16 \text { Xilgum, Lascod, Italy. }}$ 
Ahmed MHM (2018) Consequence of midline mandibular flexure on bilaterally splinted and non-splinted implant-supported mandibular full arch prosthesis with immediately loaded implants: A one year clinical study

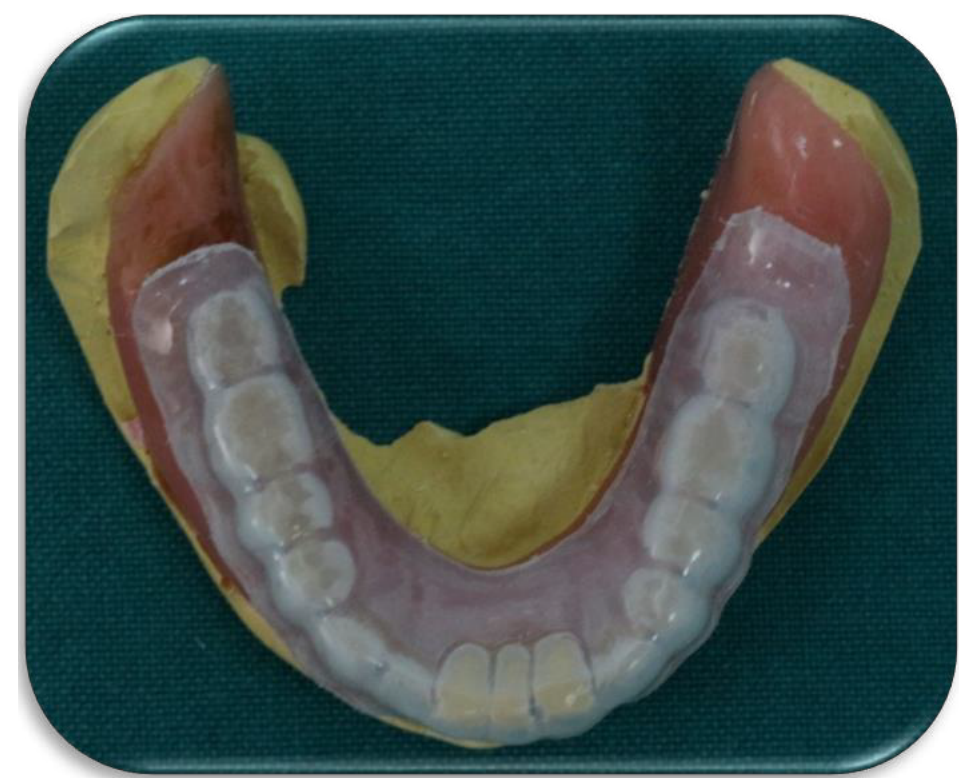

Figure 12. Pre-fabricated Clear Flexible Acrylic stent for Temporarization

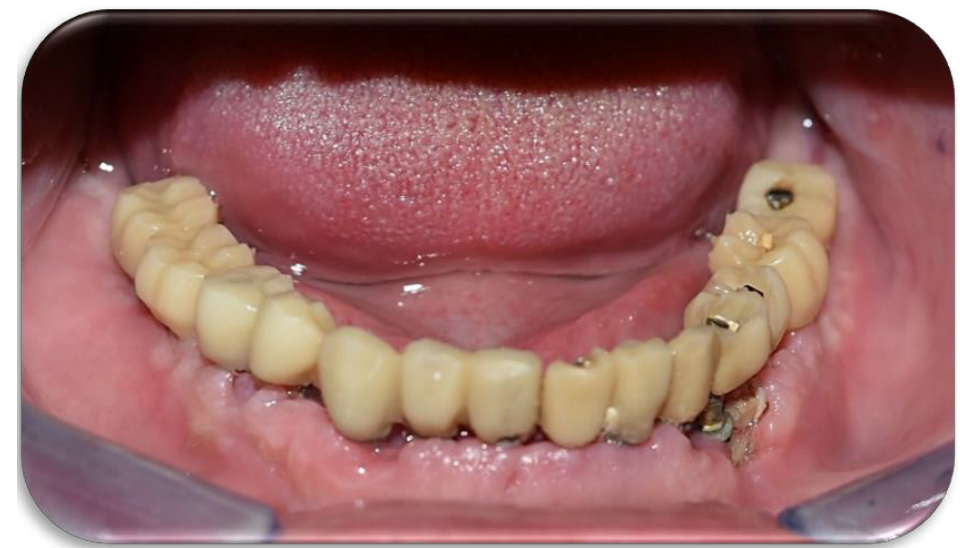

Figure 13. Direct Post-operative Temporarization

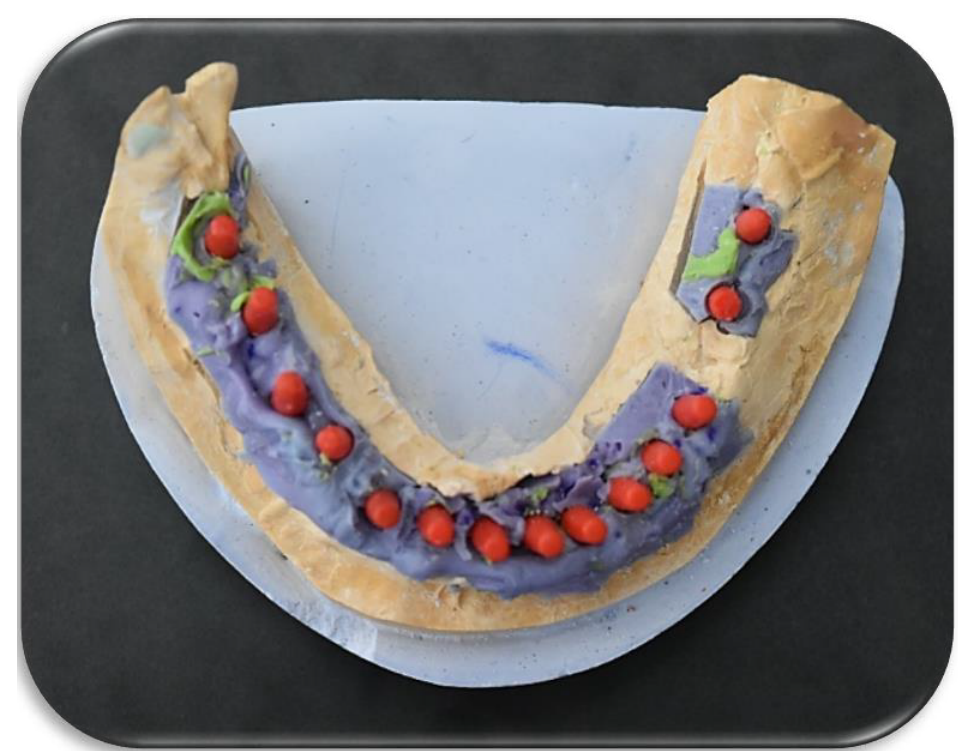

Figure 14. Pouring the Impression to obtain the Master Cast 
Ahmed MHM (2018) Consequence of midline mandibular flexure on bilaterally splinted and non-splinted implant-supported mandibular full arch prosthesis with immediately loaded implants: A one year clinical study

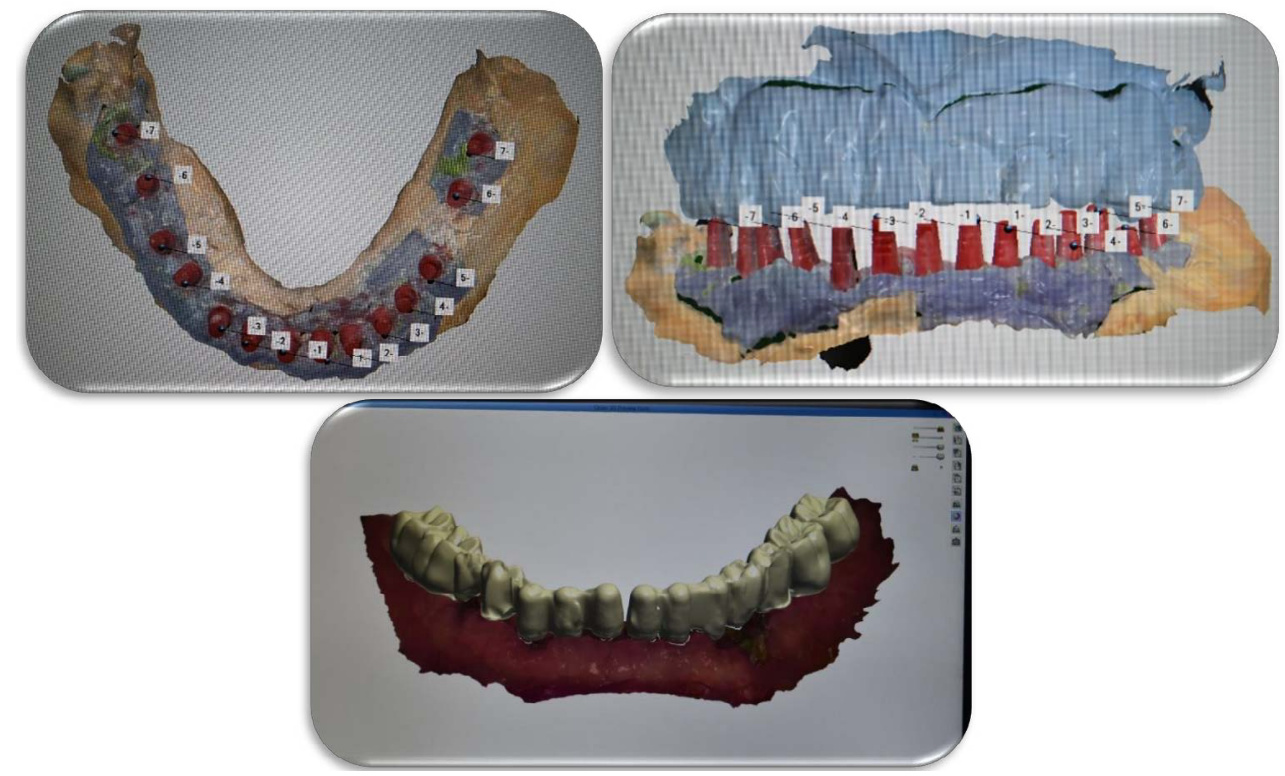

Figure 15. Scanning procedures to fabricate the final prosthesis

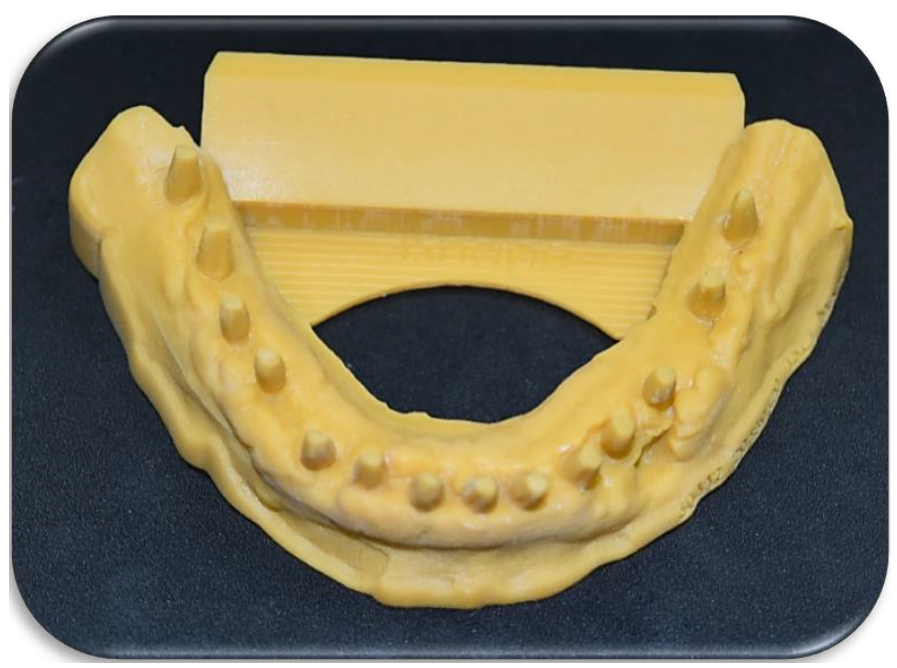

Figure 16. A 3D Printed Model

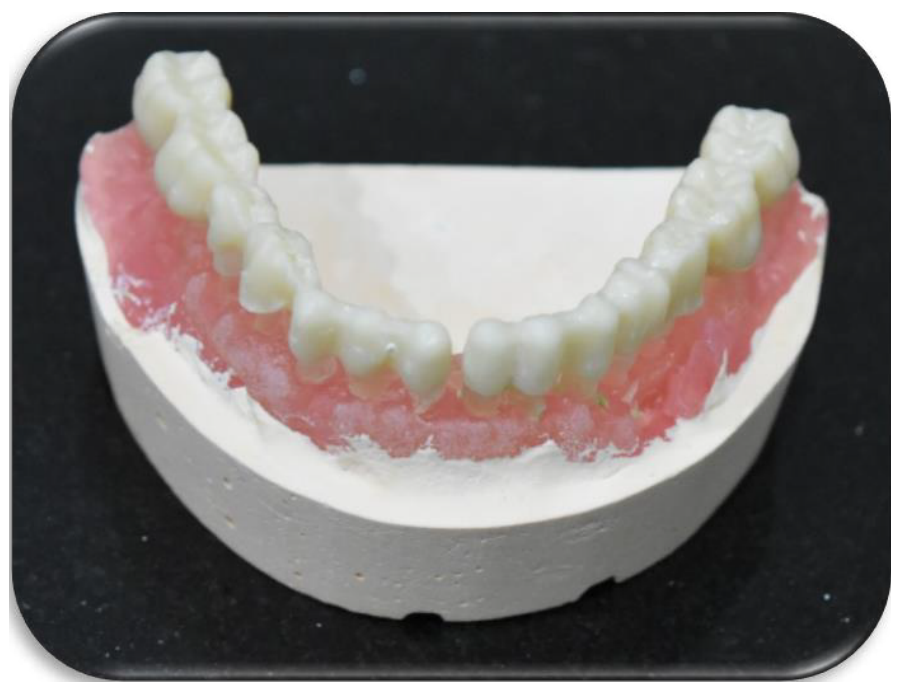

Figure 17. Construction of the final prosthesis 
Ahmed MHM (2018) Consequence of midline mandibular flexure on bilaterally splinted and non-splinted implant-supported mandibular full arch prosthesis with immediately loaded implants: A one year clinical study

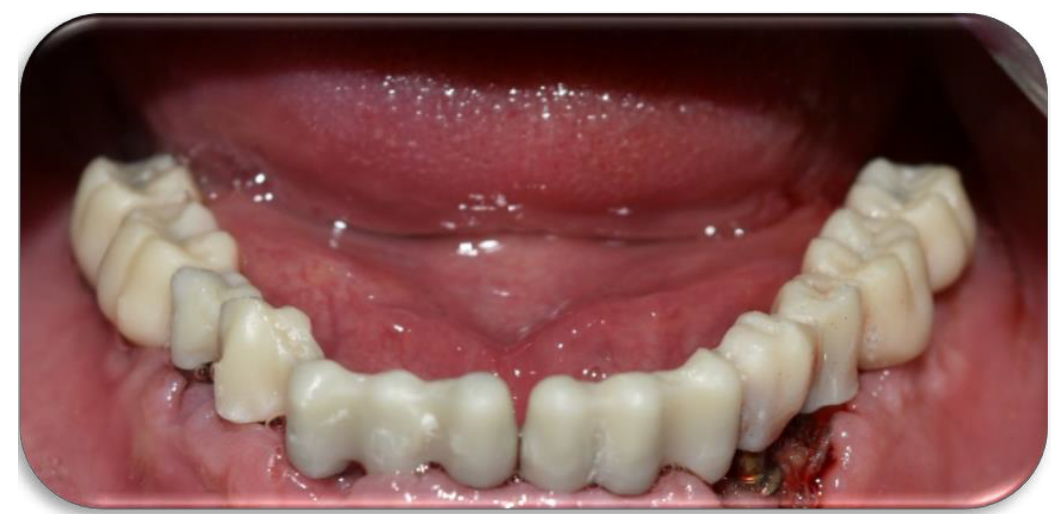

Figure 18. Try-in of the prosthesis intra-orally

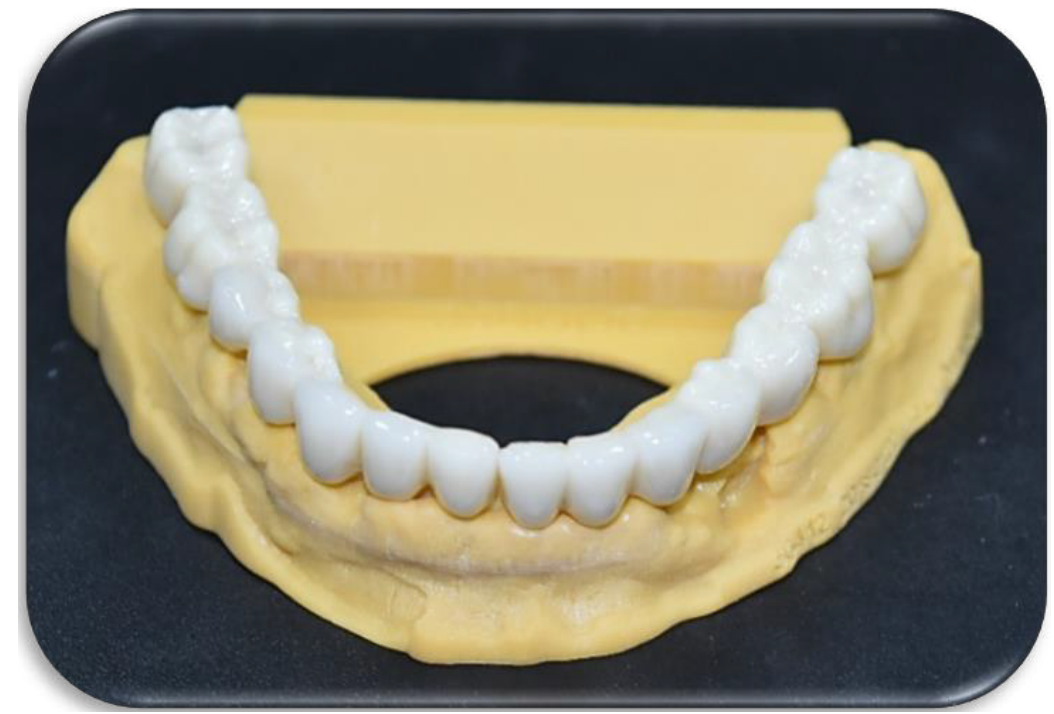

Figure 19. Final prosthesis on the cast
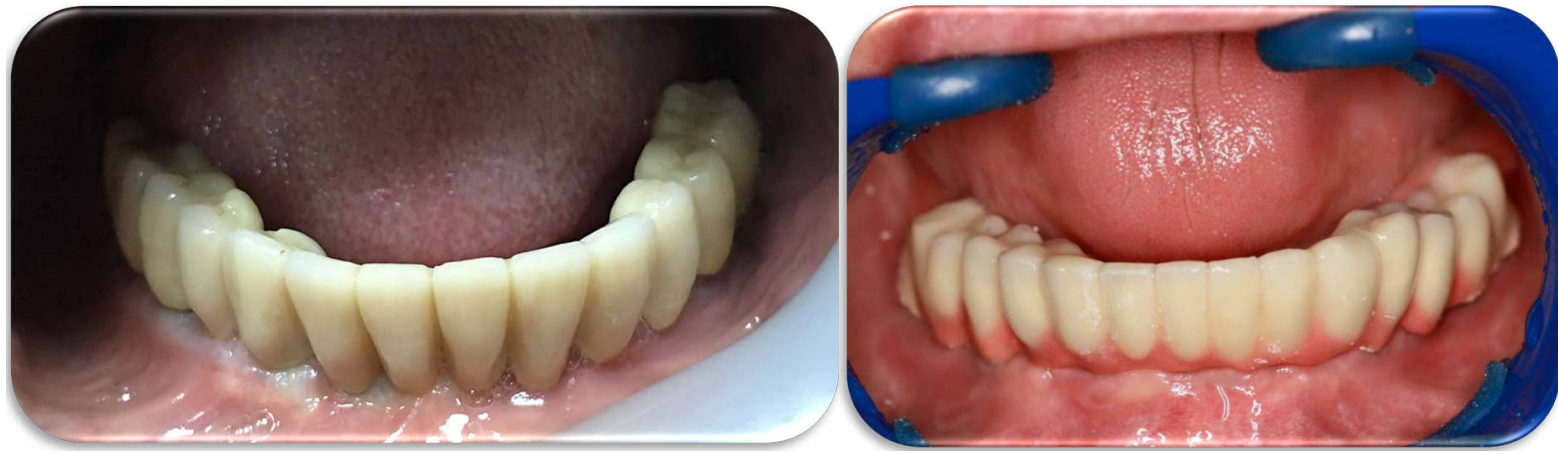

Figure 20. Final Splinted \& Non-splinted prostheses Intra-Orally

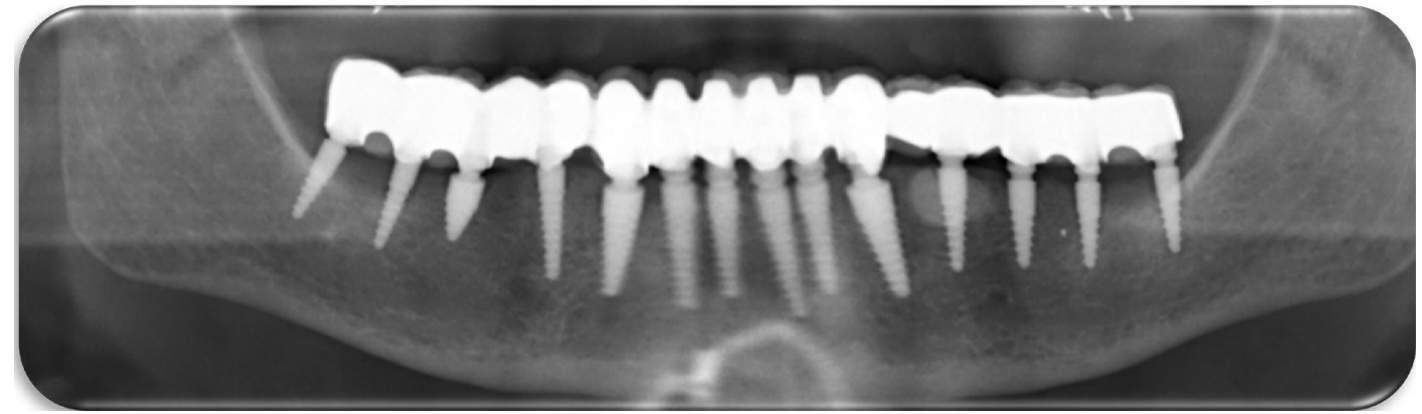

Figure 21. A Post-Operative Panoramic x-ray for the Final Prosthesis 
Ahmed MHM (2018) Consequence of midline mandibular flexure on bilaterally splinted and non-splinted implant-supported mandibular full arch prosthesis with immediately loaded implants: A one year clinical study

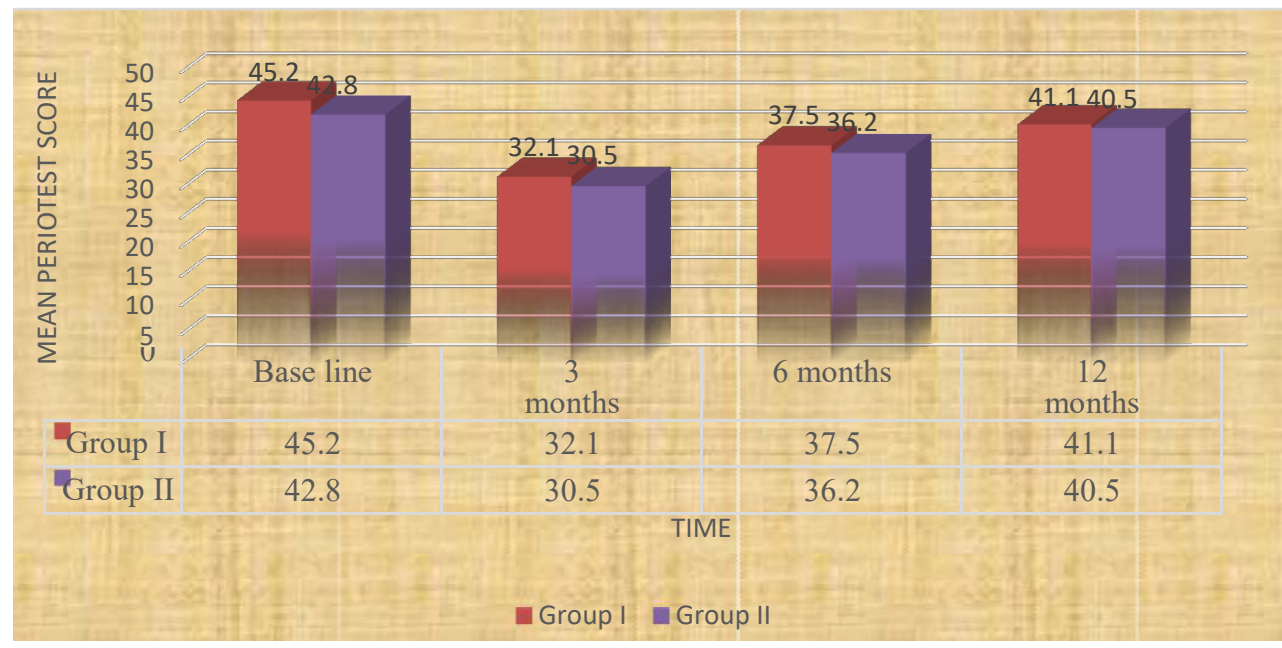

Figure 22. Bar chart representing mean Periotest scores in the two groups

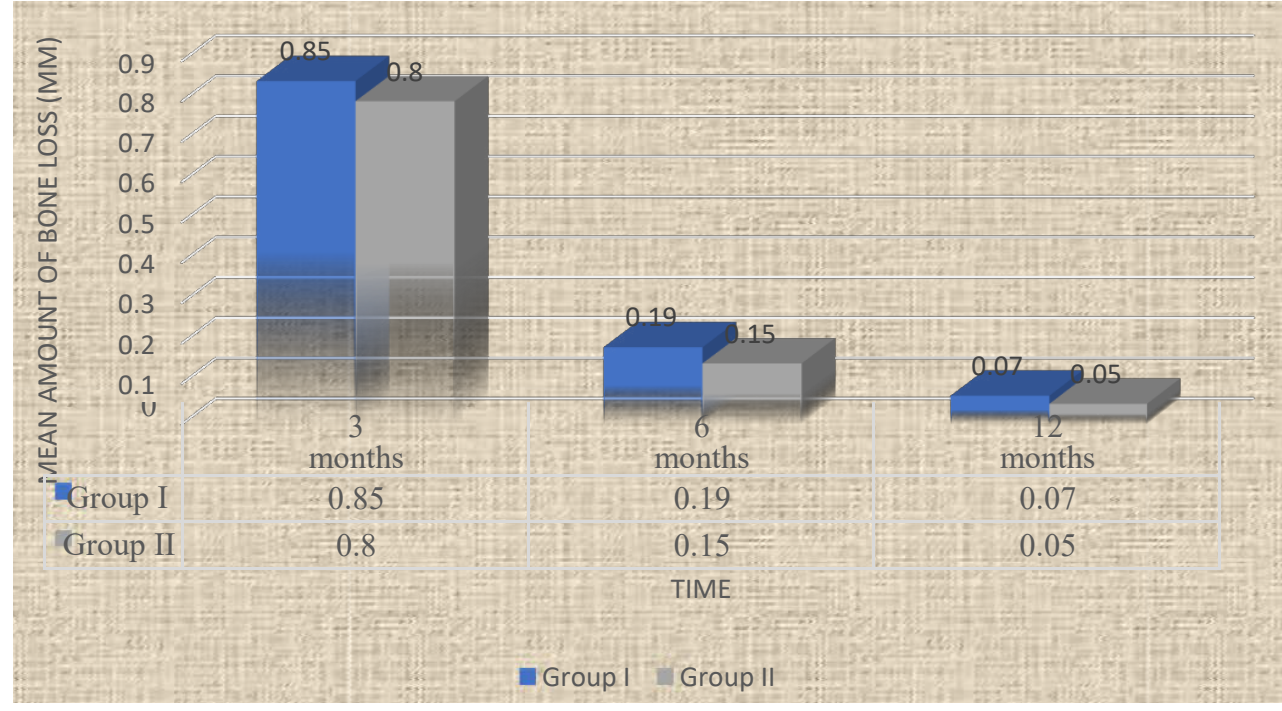

Figure 23. Bar chart representing mean amounts of bone loss in the two groups

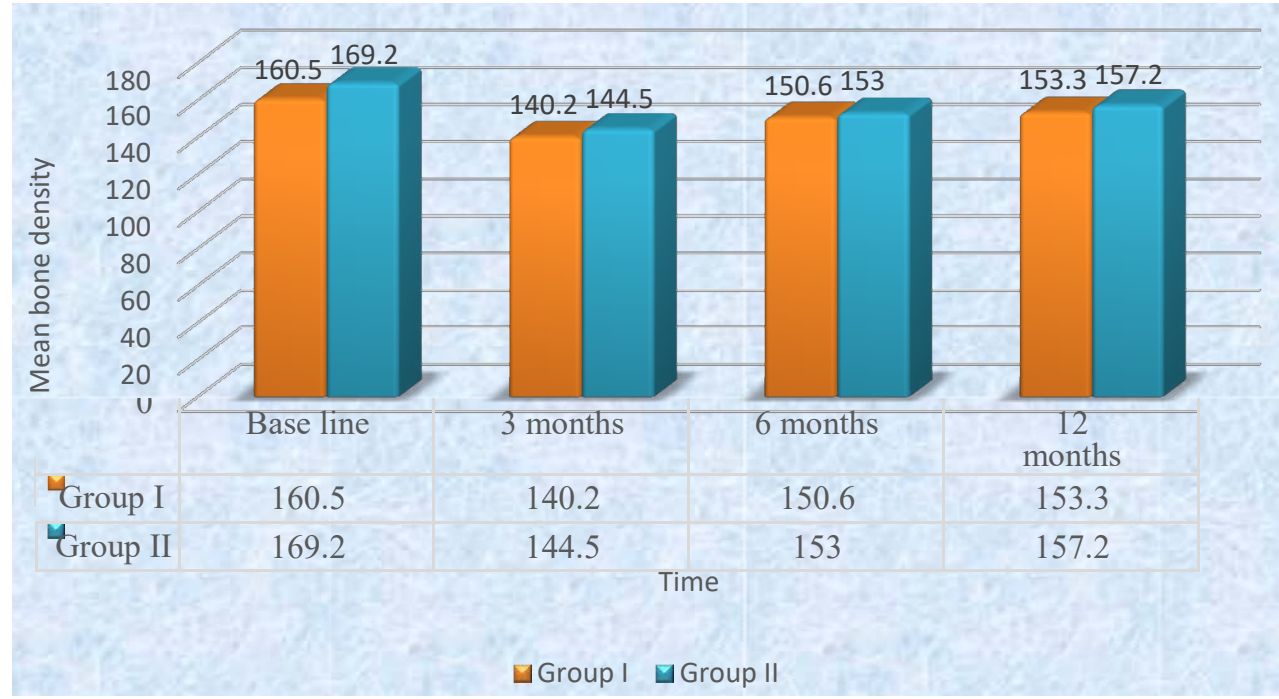

Figure 24. Bar chart representing mean bone density measurements in the two groups 


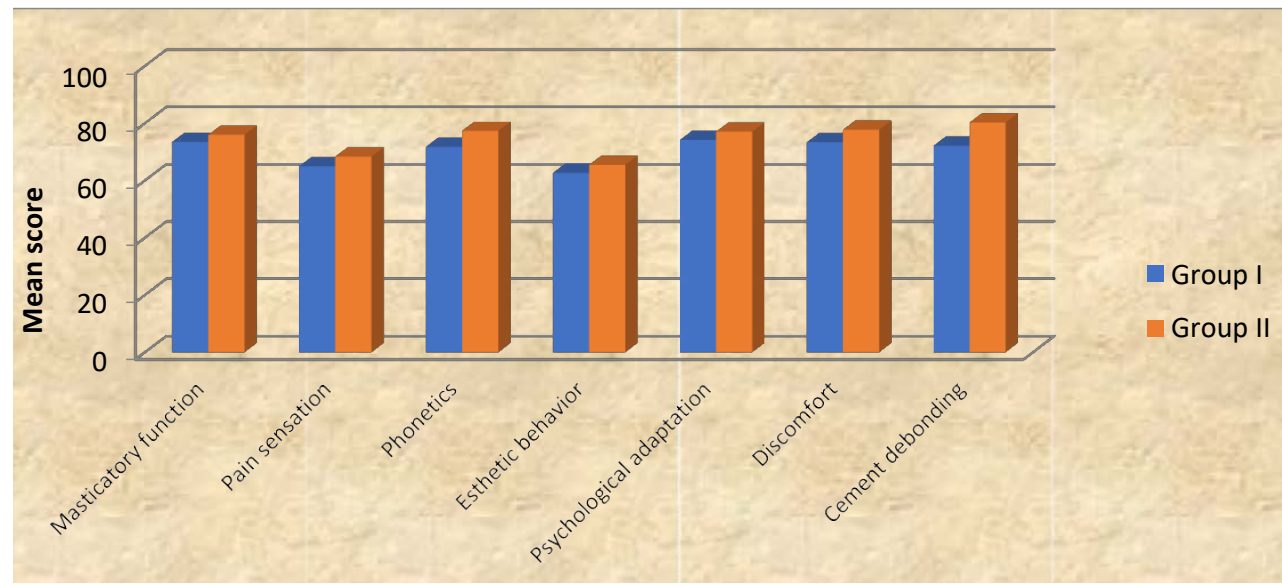

Figure 25. Bar chart representing mean satisfaction scores in the two groups

Upper cast (with its corresponding denture) \& lower casts are mounted on a semiadjustable articulator ${ }^{17}$ by the aid of a face-bow record \& an inter-occlusal centric jaw relation record.

Scanning of the impression transfers was made with laboratory scanning system ${ }^{18}$, and the core of the prosthetic crowns were fabricated utilizing a CAD/CAM laboratory system. ${ }^{19}$

Three days to the surgery, the crown cores were tried in the patient's mouth for accuracy, contour and passive fit.

An articulating paper was utilized to detect any pressure areas, which were removed (if present) by spot grinding.

The whole prosthesis assembly was then transferred to the laboratory to complete the fabrication procedures till obtaining the final zircon fixed prosthesis (fabricated to occlude in centric and in all eccentric positions) and the patient was asked for temporary cementation after 7 days of surgery.

After three months, the patients were checked for maintaining the oral hygiene measures and then starting the restorative phase of treatment. A post-operative Panoramic radiograph was made for the implant to ensure osseointegration.

Final cementation of the cement-retained prosthesis was then performed. Meanwhile, the clinical measurements (utilizing the Periotest) as well as the radiographic readings (utilizing the Digora computerized system) were performed in the same manner to obtain the second readings.

The patient was then asked for a recall appointment after three months (i.e. six months from implant placement), where the same procedures of obtaining the clinical as well as the radiographic readings were performed without any adjustments made to the crown.

The patient was then asked for a recall appointment after six months (i.e. twelve months from implant placement), where the same procedures of obtaining the clinical as well as the radiographic readings were performed.

Patients' satisfaction was also measured (the day following prosthesis placement) utilizing a customized chart of question, where; Patients answered a series of questions to evaluate the IMPLANTSUPPORTED PROETHESIS in Both Groups (7 POINTS SCALE)

17 Bio. Art articulator 5000, Brazil

18 Carestream, Italy

19 Exocad software in terms of; masticatory function, pain sensation, phonetics, esthetic behaviour, psychological adaptation, presence of any discomfort and cement debonding. Finally, each patient had to give a number representing his General satisfaction $(90-100 \%=$ Excellent, $80-89 \%=$ very good, $7079 \%=$ good, $60-69 \%=$ fair, $50-59 \%=$ working, BELOW $50 \%=$ not satisfied) Regarding the previous questions.

* Patients of both groups were asked for recall appointments 3 months, 6 months and 12 months following prosthesis insertion also for assessment of the patients' satisfaction.

- All the results were calculated, tabulated and then statistically analysed.

\section{Results}

\section{Statistical analysis}

Numerical data were explored for normality by checking the data distribution and using Kolmogorov-Smirnov and Shapiro-Wilk tests. Periotest and bone density data showed normal (parametric) distribution while bone loss and satisfaction scores data showed nonnormal (nonparametric) distribution. Data were presented as mean and standard deviation values.

For parametric data; repeated measures ANOVA test was used to compare between the two groups as well as to study the changes by time within each group. Bonferroni's post-hoc test was used for pair-wise comparisons when ANOVA test is significant. For nonparametric data; Mann-Whitney U test was used to compare between the two groups.

The significance level was set at $\mathrm{P} \leq 0.05$. Statistical analysis was performed with IBM ${ }^{\oplus 20}$ SPSS $^{\otimes 21}$ Statistics Version 20 for Windows.

\section{Periotest measurements}

Comparison between the two groups revealed that there was no statistically significant difference between the two groups through the whole study period.

\section{Regarding the changes by time within each group;}

From base line to 3 months; there was a statistically significant decrease in mean Periotest measurements.

$20{ }^{\circledR}$ IBM Corporation, NY, USA

$21{ }^{\circledR}$ SPSS, Inc., an IBM Company 
Ahmed MHM (2018) Consequence of midline mandibular flexure on bilaterally splinted and non-splinted implant-supported mandibular full arch prosthesis with immediately loaded implants: A one year clinical study

From 3 months to 6 months; there was a statistically significant increase in mean Periotest measurements.

From 6 months to 12 months; there was non-statistically significant increase in mean Periotest measurements.

From base line to 12 months; there was non-statistically significant decrease in mean Periotest measurements.

\section{Amount of bone loss}

There was no statistically significant difference between amounts of bone loss in the two groups either after3, 6 or 12 months.

\section{Bone density measurements}

Comparison between the two groups revealed that there was no statistically significant difference between the two groups through the whole study period.

\section{Regarding the changes by time within each group}

From base line to 3 months: there was a statistically significant decrease in mean bone density measurements.

From 3 months to 6 months, there was a statistically significant increase in mean bone density.

From 6 months to 12 months; there was non-statistically significant increase in mean bone density measurements.

From base line to 12 months; there was non-statistically significant decrease in mean Periotest measurements.

\section{Patient satisfaction}

Comparison between the two groups revealed that there was no statistically significant difference between satisfaction scores in the two groups.

\section{Discussion}

\section{Discussion of methodology}

In this study, all factors that could affect the osseointegration of implants were carefully considered during patient selection and later after restoration. These factors may be biological or mechanical or both; The biological factors could be related to the patient's selection, to the steps of implant installation and to the level of oral hygiene measures followed [34].

Twelve fully edentulous, healthy patients of age ranging from 55-65 years old were included in this study to avoid any fluctuation in bone changes that might affect the obtained results.

Maintenance of good oral hygiene has a great influence on the success of this study to the extent that it has a great impact on the osseointegration process. The oral hygiene of each patient was, therefore, evaluated at the beginning of the study and then throughout the whole investigation period.

Patients exhibited adequate inter-arch space, to ensure adequate room for all the overdenture components without encroachment on the normal vertical dimension of occlusion. Patients with superior general health were only selected, to avoid the reflection of any systemic disorder on the bone condition, and hence, osseointegration [35].

Only cases with normal maxilla-mandibular relation were included in the study to avoid the effect of transmission of abnormal forces to the implants. Uncooperative patients were excluded, where only cooperative patients were included in the study to ensure their commitment to the oral hygiene measures and the regular follow up visit.

Tentative jaw relations have been made to the patients to ensure an adequate inter-arch space. Moreover, it helped in determination of ridge relationship where patients only with Angle class I were included in the study to facilitate implant insertion and avoid implants overloading [36].

Bone quality and quantity were evaluated radiographically to ensure primary stability of the implant at the time of its placement. In addition, patients with sufficient bucco-lingual width at sites of implants placement were only selected to ensure at least one $\mathrm{mm}$. thickness of bone remaining buccal and lingual to the implant after its placement [33].

Construction of a computer-guided surgical stent was carried out, to ensure accuracy of implant placement in the three dimensions as well as decreasing the human interfering factors that might affect the adjustment of implants angulation [37].

For infection control during and after implant installation, preand post-surgical antibiotics and chlorohexidine mouth wash were prescribed. All implants used were Single-piece, threaded, self-tapping, root form implants, $12 \mathrm{~mm}$ length and $3.5 \mathrm{~mm}$ width. This implant design was used to ensure primary stability during the initial healing period, as well as, increasing the contact area between the implant and the surrounding bone for better osseointegration [37].

Panoramic radiograph was the imaging modality of choice in evaluating implants osseointegration, to avoid the metallic artifacts that accompany CBCT (due to presence of multiple implants) [22]. A rubber base impression material was utilized to ensure accurate recording of fine details, and hence, obtaining an accurate restoration [25]. Varnishing the impression surfaces surrounding the analogues with Vaseline was carried out to facilitate impression removal from the stone cast and ensuring that the implant analogues remain embedded into the stone cast. The cases were followed up for one year to ensure proper evaluation of clinical \& radiographic parameters as well as patients' satisfaction throughout a suitable period of time.

\section{Discussion of results}

Oral rehabilitation with implant-supported overdentures in completely edentulous mandibles offers a wide range of treatment modalities based on the varying number of implants utilized, as well as the loading behaviors approached [38]. Medial mandibular flexure (MMF) is a mandibular deformation characterized by a decrease in the arch width during jaw opening and protrusion movements because of the functional contraction of the lateral pterygoid muscles, causing high strain in the symphysial region [39].

The MMF is measured as the decrease in the intermolar distance during the movements referred above (mandible maximum opening, middle opening and protrusion) [40]. Where, the values of maximum mandibular flexure were obtained in the models of edentulous human mandibles with the lowest values of mandibular deformation. The maximum deformations were due to the protrusion movement. The minimum mandibular flexure was found to be caused by the middle opening.

The mandible, for being part of the stomatognathic system, presents a dynamic of movement. The contraction of muscles during 
Ahmed MHM (2018) Consequence of midline mandibular flexure on bilaterally splinted and non-splinted implant-supported mandibular full arch prosthesis with immediately loaded implants: A one year clinical study

mandibular movements place the mandibular condyles closer to each other, generating stress lines in the region of the chin.

The flexure movement occurs not only in the opening and protrusion movement, it also occurs in laterality, retrusion and closing movements, but with less intensity. Many authors also observed that the mandible performs flexure when it is taken to centric relation position, but in a direction opposite to the movement of opening and protrusion, i.e., there is an increase in the width of the dental arch [40].

According to some authors there is no significant difference in medial mandibular flexure in the maximum opening between men and women, age ranges and different configurations of the mandibular arch [41]. In implant-supported fixed prostheses, an ideal biomechanical distribution of stresses at the prosthetic superstructure and implant infrastructure is of dominant importance, being affected by several factors such as correct prosthetic design and occlusal scheme, among others. Attaining less stress in an implant-supported restorative system is one of the main goals of implant treatment. The mandibular flexure must be considered an important factor because it could contribute to discomfort related to the patients' rehabilitation with a mandibular fixed implant-supported prosthesis during function [26].

Until now, there is no conclusive evidence on the degree of MMF required to cause clinical problems. Also, it is unknown the long-term clinical effect of it in oral rehabilitation. Despite the need for additional studies on the influence of the medial mandibular flexure on prosthetic treatment.

A study of stress analysis has shown that frameworks constructed with a precise and passive fit induce significantly smaller amounts of stress on the implant [23] and this could be achieved by sectioning the framework. Thus, the section of the framework could decrease the stress on the implant during functional movement of the mandible, especially when posterior implants are installed behind the mental foramen, increasing their longevity. Sectioning the prostheses into two pieces $[12,18]$ has been recommended to allow mandibular flexure of the restored mandible to come close to its natural state [11]. It has been hypothesized that these designs will minimize stress concentration in posterior and anterior implants [13]. However, despite the biomechanical advantages of these fixed implant supported prostheses designs, the aesthetic is affected by the sectioning of the final prosthesis and these sections could lead food to impact on the sectioned areas, compromising the patient's hygiene. The contraction of the lateral pterygoid muscle is the most important feature producing mandibular deformation during function and four patterns of jaw deformation were postulated: symphysial bending, dorsoventral shear, corporal rotation, and anteroposterior shear [27].

Framework Splitting into two segments at the mandibular midline might decrease the stress during symphysial bending, although it won't avoid the forces induced by corporal rotation. In the current study, the installation of posterior implants was used to decrease the lever arm, allowing greater posterior extension and increased occlusion scheme in the mandibular fixed implant-supported prosthesis, which provided a better distribution of occlusal forces and increased the prosthesis stability.

The statistically significant decrease in the mean Periotest measurements from base line to three months might be attributed to the inflammatory, resorptive and remodeling activities during the healing process.

These findings were in line with those studies which concluded that; although new implant surface and chemistry designs have shortened and improved osseointegration, the initial implant stability drop is still present and remains a challenge for future research and development [42].

Furthermore, the mechanism behind the decrease of measurements during the first three months might be related to the changes of boneimplant interface as well as the properties of the surrounding bone (i.e. the interfacial stiffness). Firstly, the tapered implant creates a lateral compression of the bone tissue during insertion and it is likely that the relaxation of the inbuilt stresses between the implant and bone occurred after implant placement, which can be considered as a decrease in scores. Secondly, it can be speculated that loading might induce microfractures in the surrounding bone. These were in line with many similar studies [43].

The statistically significant increase in the mean Periotest scores from three months to six months of prosthesis insertion, indicating the increased amount of bone formation around the implants and hence, greater implant osseointegration [44].

The statistically significant increase in the mean Periotest scores from base line to twelve months matched the time frames of bone formation and maturation around the dental implants [44]. The significant increase in the mean bone height measurements in both groups, indicating increased crestal bone resorption in the first three months compared to those measurements from six to twelve months, which might be explained by the continuous remodeling process of bone surrounding the implant resulting in bone resorption, followed by bone deposition [45].

The crestal bone resorption around implants is a well-known phenomenon occurring mostly in the initial phase of functional implant loading and considered as an immediate bone response after insertion of the implant supported prosthesis. The mean marginal bone loss in the present study from base line to twelve months is considered within accepted permissible limits occurring with most dental implants [36]. Regarding changes of bone density around the implant, it was evident that there was a significant decrease of mean values of bone density at the first 3 months. This was mainly attributed to the surgical trauma during implant surgery. In addition to, the precautions given to the patient to maintain soft diet during the initial phase of treatment [46].

The statistically significant increase in the bone density measurements in periods from three to six months, indicating favorable bone reaction to the applied forces that were within the physiologic limit tolerated by the bone and hence, favorable progress of the osseointegration process.

During the recall periods of all patients, there were no complaints from the installed implant and all the patients followed the oral hygiene instructions to avoid any harmful effect which might influence the results of this study [32].

Several studies reported that, the patients' quality of life had been improved by the increased retention and stability of their implantsupported fixed prostheses [35]. The non-statistically significant difference in patients' satisfaction between the two groups throughout the whole study period, showed that whatever the utilized Splinting technique is, would not affect the efficiency of the used prosthesis [36]. The results of the present clinical investigation confirmed the null hypothesis previously established (Table 1-3).

\section{Conclusion}

Within the limits of this clinical research study, concerning the relatively small sample size, it could be conservatively determined that: 
Ahmed MHM (2018) Consequence of midline mandibular flexure on bilaterally splinted and non-splinted implant-supported mandibular full arch prosthesis with immediately loaded implants: A one year clinical study

Table 1. The mean, standard deviation (SD) values and results of repeated measures ANOVA test for comparison between Periotest scores in the two groups as well as changes by time within each group

\begin{tabular}{|c|c|c|c|c|c|}
\hline \multirow{2}{*}{$\begin{array}{l}\text { Group } \\
\text { Time }\end{array}$} & \multicolumn{2}{|c|}{ Group I } & \multicolumn{2}{|c|}{ Group II } & \multirow{2}{*}{$\begin{array}{c}P \text {-value } \\
\text { (Between groups) }\end{array}$} \\
\hline & Mean & SD & Mean & SD & \\
\hline Base line & $45.2^{\mathrm{A}}$ & 7.2 & $42.8^{\mathrm{A}}$ & 9.1 & 0.775 \\
\hline 3 months & $32.1^{\mathrm{C}}$ & 9.5 & $30.5^{\mathrm{C}}$ & 8.7 & 0.582 \\
\hline 6 months & $37.5^{\mathrm{B}}$ & 10.2 & $36.2^{\mathrm{B}}$ & 11.5 & 0.681 \\
\hline 12 months & $41.1^{\mathrm{AB}}$ & 8.5 & $40.5^{\mathrm{AB}}$ & 7.6 & 0.605 \\
\hline $\begin{array}{c}P \text {-value } \\
\text { (Within group) }\end{array}$ & \multicolumn{2}{|c|}{$<0.001^{*}$} & \multicolumn{2}{|c|}{$<0.001^{*}$} & \\
\hline
\end{tabular}

*Significant at $P \leq 0.05$, Different superscripts in the same column are statistically significantly different.

Table 2. The mean, standard deviation (SD) values and results of Mann-Whitney U test for comparison between amounts of bone loss in the two groups.

\begin{tabular}{|c|c|c|c|c|}
\hline \multirow{2}{*}{$\begin{array}{c}\text { Group } \\
\text { Time }\end{array}$} & Mean & Group I & \multicolumn{2}{|c|}{ Group II } \\
\hline 3 months & 0.85 & 0.23 & Mean & SD \\
\hline (B) months & 0.19 & 0.11 & 0.80 & 0.20 \\
\hline 12 months & 0.07 & 0.04 & 0.15 & 0.647 \\
\hline
\end{tabular}

*Significant at $P \leq 0.05$

Table 3. The mean, standard deviation (SD) values and results of repeated measures ANOVA test for comparison between bone density measurements in the two groups as well as changes by time within each group

\begin{tabular}{|c|c|c|c|c|c|}
\hline \multirow{2}{*}{$\begin{array}{c}\text { Group } \\
\text { Time }\end{array}$} & \multicolumn{2}{|c|}{ Group I } & \multicolumn{2}{c|}{ Group II } \\
\hline Base line & Mean & SD & Mean & SD \\
\hline 3 months & $160.5^{\mathrm{A}}$ & 13.3 & $169.2^{\mathrm{A}}$ & 15.6 \\
\hline 6 months & $140.2^{\mathrm{C}}$ & 16.5 & $144.5^{\mathrm{C}}$ & 13.6 \\
\hline 12 months & $150.6^{\mathrm{B}}$ & 21.7 & $153.0^{\mathrm{B}}$ & 18.7 \\
\hline $\begin{array}{c}P \text {-value } \\
\text { (Within group) }\end{array}$ & $153.3^{\mathrm{B}}$ & 31.5 & 157.2 & 0.152 \\
\hline
\end{tabular}

*Significant at $P \leq 0.05$, Different superscripts in the same column are statistically significantly different

-This study was conducted to compare the effect of effect of midline mandibular flexure on bilaterally splinted \& non-splinted (segmented) cement-retained, immediately-loaded mandibular prosthesis.

- Twelve patients were cautiously selected from the outpatient clinic of the Removable Prosthodontic department, Faculty of Dentistry, Cairo University, with edentulous arches. Patients were randomly divided into two groups; First group (of six patients) had received Implant-supported, fully-splinted cement-retained full arch prosthesis with immediate functional loading protocol, while Second group (of six patients) had received Implant supported, non-splinted (segmented) cement-retained full arch prosthesis with immediate functional loading protocol.

The clinical \& the radiographic outcomes of the Implants supporting the cement retained full arch superstructure, had been calibrated at time of implants insertion, 3 months, 6 months and 12 months respectively. In addition, patients' satisfaction was also measured utilizing a customized chart of question (A seven-point scale).

\section{From the results of this study, the following conclusions} could be achieved

- There was non-significant effect on both types of prosthesis and hence, splinted or nonsplinted implant-supported prostheses could be used successfully with little superiority of segmentation in midline region. In addition,

- Patients were completely satisfied with their implant-supported immediately-loaded prostheses, whatever splinting protocol utilized.
- Both modalities presented a workable treatment option for supporting a cementretained mandibular prosthesis.

- Enhanced clinical investigations with larger sample sizes and over longer periods of time, are still needed.

\section{Conflict of interest}

This clinical study was self-funded by the authors, with no conflict of interest.

\section{References}

1. El-Sheikh AM, Abdel-Latif HH, Howell PGT, Hobkir JA (2007) Midline mandibular deformation during non-masticatory functional movements in edentulous subjects with dental implants, Int J Oral Maxillofac Implants 22: 243-248.

2. Paleari AG, Presoto CD, Vasconcelos JA, Nunes Reis JM, Pinelli LA, et al. (2015) An alternative technique for fabrication of frameworks in an immediate loading implant fixed mandibular prosthesis, Case Rep Dent 2015:102189. [Crossref]

3. Nokar S, Naini RB (2005) The effect of superstructure design on stress analysis, Int $J$ Oral Maxillofac Implants 20: 533_539

4. Attard NJ, Zarb GA (2005) Immediate and early implant loading protocols: a literature review of clinical studies. J Prosthet Dent 94:242-258. [Crossref]

5. Becker W, Goldstein M (2008) Immediate implant placement: treatment planning and surgical steps for successful outcome. Periodontol 2000 47: 79-89. [Crossref]

6. Bernardes SR, de Araujo CA, Neto AJ, Simamoto Junior P, das Neves FD (2009) Photoelastic analysis of stress patterns from different implant-abutment interfaces. Int $J$ Oral Maxillofac Implants 24: 781-789. [Crossref]

7. Blanes RJ (2009) To what extent does the crown-implant ratio affect the survival and complications of implant-supported reconstructions? A systematic review. Clin Oral Implants Res 20 (suppl 4): 67-72. [Crossref]

8. Law C, Bennani V, Lyons K, Swain M (2014) Influence of implant framework and mandibular flexure on the strain distribution on a Kennedy class II mandible restored with a long-span implant fixed restoration: a pilot study, J Prosthet Dent 112: 31-37. [Crossref] 
Ahmed MHM (2018) Consequence of midline mandibular flexure on bilaterally splinted and non-splinted implant-supported mandibular full arch prosthesis with immediately loaded implants: A one year clinical study

9. Fischman B (1990) The rotational aspect of mandibular flexure. J Prosthet Dent 64: 483-485. [Crossref]

10. Zarone F, Apicella A, Nicolais L, Aversa R, Sorrentino R (2003) Mandibular flexure and stress build-up in mandibular full-arch fixed prostheses supported by osseointegrated implants. Clin Oral Implants Res 14: 103-114. [Crossref]

11. Capelli M, Zuffetti F, Del Fabbro M, Testori T (2007) Immediate rehabilitation of the completely edentulous jaw with fixed prostheses supported by either upright or tilted implants: a multicenter clinical study. Int J Oral Maxillofac Implants 22:639-644. [Crossref]

12. Misch CE (2005) Mandibular Full-Arch Implant Fixed Prosthetic Options: În: Misch C.E. Dental Implant Prosthetics, Elsevier Mosby, St. Louis, 256-264.

13. Crespi R, Vinci R, Cappare P, Romanos GE, Gherlone E (2012) A clinical study of edentulous patients rehabilitated according to the "all on four" immediate function protocol. Int J Oral Maxillofac Implants 27:428-434. [Crossref]

14. Degidi M, Nardi D, Piattelli A (2009) Immediate rehabilitation of the edentulous mandible with a definitive prosthesis supported by an intraorally welded titanium bar. Int J Oral Maxillofac Implants 24: 342-347. [Crossref]

15. Esposito M, Grusovin MG, Willings M, Coulthard P, Worthington HV (2007) The effectiveness of immediate, early, and conventional loading of dental implants: a Cochrane systematic review of randomized controlled clinical trials. Int J Oral Maxillofac Implants 22:893-904. [Crossref]

16. Francetti L, Rodolfi A, Barbaro B, Taschieri S, Cavalli N, et al. (2015) Implant success rates in full-arch rehabilitations supported by upright and tilted implants: retrospective investigation with up to five years of follow-up. $J$ Periodontal Implant Sci 5:210-215. [Crossref]

17. Gallucci GO, Benic GI, Eckert SE, et al. (2014) Consensus statements and clinica recommendations for implant loading protocols. Int J Oral Maxillofac Implants 29(suppl): 287-290. [Crossref]

18. Jacques LB, Moura MS, Suedam V, Souza EA, Rubo JH (2009) Effect of cantilever length and framework alloy on the stress distribution of mandibular-cantilevered implant-supported prostheses. Clin Oral Implants Res 20:737-741. [Crossref]

19. Sivaraman K, Chopra A, Venkatesh SB (2016) Clinical importance of median mandibular flexure in oral rehabilitation: a review $J$ Oral Rehabil 43; 215-225. [Crossref]

20. Kwon T, Bain PA, Levin L (2014) Systematic review of short- (5-10 years) and longterm (10 years or more) survival and success of full-arch fixed dental hybrid prostheses and supporting implants. J Dent 42:1228-1241. [Crossref]

21. Millen C, Bragger U, Wittneben JG (2015) Influence of prosthesis type and retention mechanism on complications with fixed implant-supported prostheses: a systematic review applying multivariate analyses. Int J Oral Maxillofac Implants 30:110-124. [Crossref]

22. Paniz G, Stellini E, Meneghello R, Cerardi A, Gobbato EA, et al. (2013) The precision of fit of cast and milled full-arch implant-supported restorations. Int J Oral Maxillofac Implants 28:687-693. [Crossref]

23. Zarb GA, Hobkirk J, Eckert S, Jacob R, Prosthodontic Treatment for Edentulous Patients: Complete Dentures and Implant-Supported Prostheses, Mosby, St. Louis, Mo, USA, 13th edition, 2013

24. Chrcanovic BR, Albrektsson T, Wennerberg A (2015) Dental implants inserted in fresh extraction sockets versus healed sites: a systematic review and meta-analysis. J Dent 43:16-41. [Crossref]

25. Law C, Bennani V, Lyons K, Swain M (2012) Mandibular flexure and its significance on implant fixed prostheses: a review, J Prosthodont 21: 219-224. [Crossref]

26. Castellon P, Blatz MB, Block MS, Finger IM, Rogers B (2004) Immediate loading of dental implants in the edentulous mandible. J Am Dent Assoc 135:15431549.

27. Krennmair S, Weinlander M, Forstner T, Krennmair G, Stimmelmayr M (2016) Factors affecting peri-implant bone resorption in four Implant supported mandibular fullarch restorations: a 3-year prospective study. J Clin Periodontol 43:92101. [Crossref]
28. Patzelt SB, Spies BC, Kohal RJ (2015) CAD/CAM-fabricated implant-supported restorations: a systematic review. Clin Oral Implants Res 26(suppl 11):7785. [Crossref]

29. Naini RB, Nokar S (2009) Three-dimensional finite element analysis of the effect of 1-piece superstructure on mandibular flexure, Implant Dent 18: 428-437. [Crossref]

30. Penarrocha-Diago MA, Maestre-Ferrin L, Demarchi CL, Penarrocha-Oltra D, et al (2011) Immediate versus nonimmediate placement of implants for full-arch fixed restorations: a preliminary study. J Oral Maxillofac Surg 69:154-159. [Crossref]

31. Soydan SS, Cubuk S, Oguz Y, Uckan S (2013) Are success and survival rates of early implant placement higher than immediate implant placement? Int J Oral Maxillofac Surg 42:511-515. [Crossref]

32. Strassburger C, Kerschbaum T, Heydecke G (2006) Influence of implant and conventional prostheses on satisfaction and quality of life: A literature review. Part 2: Qualitative analysis and evaluation of the studies. Int J Prosthodont 19:339-348. [Crossref]

33. Wong NY (2016) Future Trends in Implant Dentistry: Digitally Guided Surgery and Prosthetics. Dent Today 35: 86, 88, 90-91. [Crossref]

34. Greco GD, Jansen WC, Landre Junior J, Seraidarian PI (2009) Stress analysis on the freeend distal extension of an implant-supported mandibular complete denture. Braz Oral Res 23:182-189. [Crossref]

35. Vogl S, Stopper M, Hof M, Wegscheider WA, Lorenzoni M (2015) Immediate occlusal versus non-occlusal loading of implants: a randomized clinical pilot study. Clin Implant Dent Relat Res 17:589-597.

36. Weinstein R, Agliardi E, Fabbro MD, Romeo D, Francetti L (2012) Immediate rehabilitation of the extremely atrophic mandible with fixed full-prosthesis supported by four implants. Clin Implant Dent Relat Res 14: 434-441. [Crossref]

37. Grandi T, Guazzi P, Samarani R, Grandi G (2012) Immediate loading of four (Allon-4) post-extractive implants supporting mandibular cross-arch fixed prostheses 18 month follow-up from a multicenter prospective cohort study. Eur J Oral Implantol 5:277-285.

38. Agliardi E, Clerico M, Ciancio P, Massironi D (2010) Immediate loading of full-arch fixed prostheses supported by axial and tilted implants for the treatment of edentulous atrophic mandibles. Quintessence Int 41:285-293. [Crossref]

39. Chiapasco M (2004) Early and immediate restoration and loading of implants in completely edentulous patients. Int J Oral Maxillofac Implants. 19(suppl):7691. [Crossref]

40. El-Sheikh AM, Abdel-Latif HH, Howell PG, Hobkirk JA (2007) Midline mandibula deformation during non-masticatory functional movements in edentulous subjects with dental implants. Int. J Oral Maxillofac Implants. 22:243-8.

41. Al-Sukhun J, Helenius M, Lindqvist C, Kelleway J (2007) Biomechanics of the mandible part I: Measurement of mandibular functional deformation using customfabricated displacement transducers, J Oral Maxillofac Surg 64: 1015-1022. [Crossref]

42. Wong N (2016) Predictable immediate implant prosthetics using guided surgery and guided prosthetics: a case report. Oral Health 106.1 (2016): 66-78.

43. Satoh T, Maeda Y, Komiyama Y, Biomechanical rationale for intentionally inclined implants in the posterior mandible using 3D finite element Tamilnadu Dr. M.G.R Medical University; 2005.

44. Drago CJ, Lazzara RJ (2006) Immediate occlusal Loading of Osseotite implants in mandibular edentulous patients: a prospective observational report with 18-month data J Prosthodont 15:187-194. [Crossref]

45. Del Fabbro M, Ceresoli V (2014) The fate of marginal bone around axial vs. tilted implants: a systematic review. Eur J Oral Implantol 7 Suppl 2: S171-189. [Crossref]

46. AlHelal A, AlBader B, Kattadiyil MT, Garbacea A, Proussaefs P (2017) CAD-CAM implant-supported fixed complete dental prosthesis with titanium milled molars: A clinical report. J Prosthet Dent 117:463-469. [Crossref]

Copyright: $\bigcirc 2018$ Ahmed MHM. This is an open-access article distributed under the terms of the Creative Commons Attribution License, which permits unrestricted use, distribution, and reproduction in any medium, provided the original author and source are credited. 